\title{
Mediastinum Lymph Node Metastasis with Superior Vena Cava Syndrome
}

\author{
Takeshi Kusunoki ${ }^{1, *}$, Hirotomo Homma ${ }^{1}$, Yoshinobu Kidokoro ${ }^{1}$, Akihisa Yoshikawa ${ }^{1}$, Kumiko Tanaka ${ }^{1}$, Satoko Kubo ${ }^{1}$, and Ryo \\ Wada ${ }^{2}$
}

${ }^{1}$ Department of Otorhinolaryngology, Juntendo University of Medicine, Shizuoka Hospital, Japan

${ }^{2}$ Department of Pathology, Juntendo University of Medicine, Shizuoka Hospital, Japan

*Corresponding authors: Takeshi Kusunoki, Department of Otorhinolaryngology, Juntendo University of Medicine, Shizuoka Hospital, 1129 Nagaoka Izunokuni-shi, Shizuoka 410-2295, Japan, Fax: +81-55-948-5088; E-mail: ttkusunoki001@aol.com

Received: 19 May, 2021 | Accepted: 23 Jun, 2021 | Published: 02 Jul, 2021

Citation: Kusunoki T, Homma H, Kidokoro Y, Yoshikawa A, Tanaka K, et al. (2021) Mediastinum Lymph Node Metastasis with Superior Vena Cava Syndrome. J Clin Case Stu 6(2): dx.doi.org/10.16966/2471-4925.225

Copyright: (C) 2021 Kusunoki T, et al. This is an open-access article distributed under the terms of the Creative Commons Attribution License, which permits unrestricted use, distribution, and reproduction in any medium, provided the original author and source are credited.

\section{Abstract}

Background: Superior Vena Cava Syndrome (SVCS) is an increase in the upper body venous pressure caused by obstruction or stenosis of the Superior Vena Cava (SVC), which causes congestion of the head, face, upper limbs, neck and upper body. The main etiology has been reported to be tumors of the lungs or mediastinum, and aortic aneurysm.

Case report: In this study, we experienced a case of SVCS due to mediastinum lymph node metastasis, our patient was an 83-year-old Japanese man. His history included exposure to nuclear bombing when a child in elementary school. At the time of the diagnosis of SVCS caused by mediastinum lymph node metastasis, he had received follow-up for a lung tumor, hormone therapy for prostate cancer, and postoperative cervical lymph node metastasis of the thyroid cancer. The general condition was poor and we could not perform a mediastinum lymph node biopsy. Radiation therapy was performed for this case and, once the facial edema was reduced, he died from an increase of heart failure one month after the end of radiotherapy.

Conclusion: After cancer treatment or following patients with cancer-carrier conditions, it is necessary to keep in mind the possibility of SVCS due to longitudinal lymph node metastasis, as well as chest and mediastinum tumors, if edema of the face and upper limbs appears.

Keywords: Mediastinum lymph node metastasis; Superior vena cava syndrome; Cardiac fluid retention; PET-CT

\section{Introduction}

Superior vena cava (SVC) is known to be susceptible to pressure from surrounding tissues due to various conditions such as its anatomical position, histological features of thin walls, and low blood pressure in the vassal canal [1]. Superior vena cava syndrome (SVCS) is a generic term for a syndrome in which compression or obstruction of the SVC impairs the return of venous blood to the heart and causes venous depression in the head, neck, chest, and upper limbs [2]. In the etiology of SVCS, most cases (85\%) occur in the presence of malignant disease, particularly bronchogenic carcinomas, lymphomas and metastatic tumors [3]. We experienced a case of SVCS due to mediastinum lymph node metastasis and report the clinical findings, images, cytology, treatment, progress and various considerations.

\section{Case Report}

\section{Patient}

83 year's old, male, Japanese.

\section{Chief complaint}

Facial edema.

\section{Past history}

When a child in school, the patient was exposed to an atomic bomb.

\section{Course}

At the age of 73 (20XX-10 years), a right thyroid tumor was pointed out by CT during examination of the right pulmonary multiple nodules for respiratory surgery.

Previously, otolaryngologists performed right thyroid lobectomy and right lymph node dissection. Postoperative pathological examination revealed thyroid papillary carcinoma with right cervical lymph node metastasis (T2NIbM0). For the respiratory surgery, biopsy of the lung nodules was recommended to the patient to determine whether it was primary lung cancer or lung metastasis from the thyroid cancer. However, he did not agree to the lung biopsy. At the age of 75 (20XX-8 years), it was pointed out by the urology department that he had prostate cancer with multiple vertebrae involvement. Therefore, he received hormone treatment and maintained good control. Two weeks before, he noticed edema of the face and fingers (Figure 1). Echo examination showed findings of multiple metastases from a lymph node at the right side of the neck. Fine needle aspiration cytology of this lymph node revealed cervical lymph node metastasis from the thyroid carcinoma. In the blood test, the thyroid function was almost normal, but thyroglobulin showed a high value of $839 \mathrm{ng}$ (normal range: $0-33 \mathrm{ng} / \mathrm{ml}$ ). PET-CT and contrast enhanced CT showed enlarged mediastinum lymph nodes infiltrating the SVC in addition to lymph node metastasis at the right 

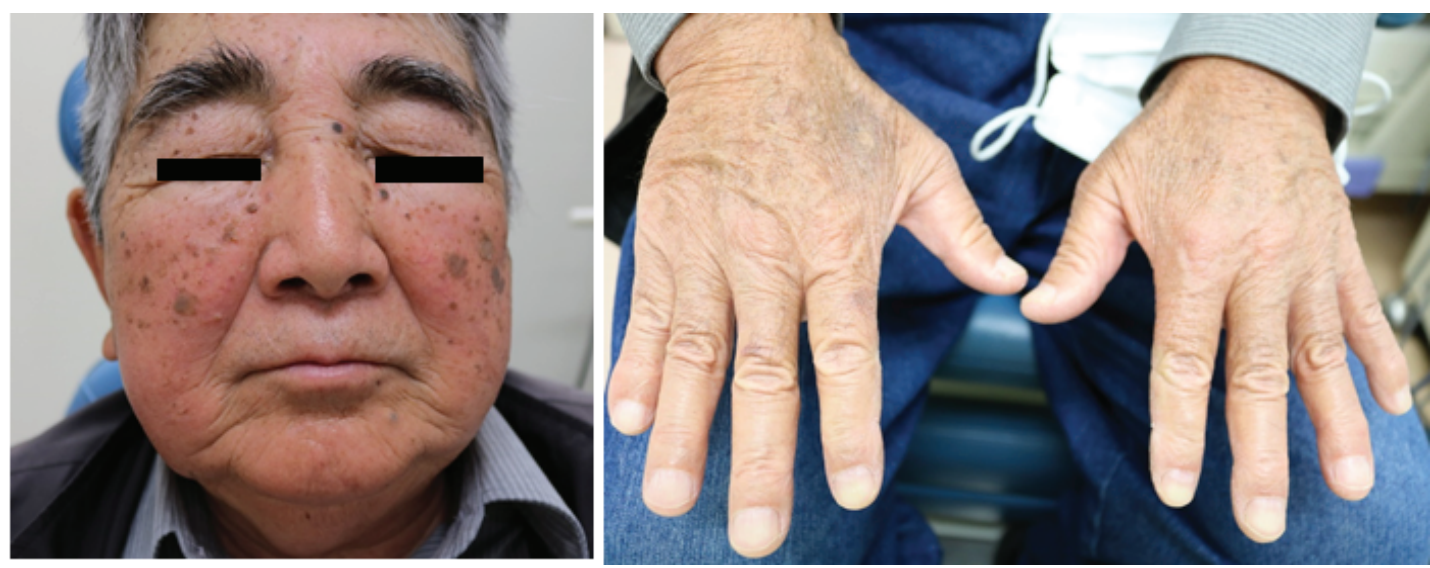

Figure 1: left: The patient's face (left) and fingers (right) were edematous.

side of the neck. Moreover, cardiac fluid retention and pleural fluid were observed (Figure 2).

These enlarged medastinum lymph nodes were considered to represent possible metastasis from lung cancer or prostate cancer, in addition to thyroid cancer. In order to determine the origin of the medastinum lymph node metastasis, puncture aspiration cytological examination of the cardiac sac fluid was performed and diagnosed as adenocarcinoma (Figure 3). Since his general condition was poor, he could not undergo biopsy of the mediastinum lymph nodes. He did not wish to be examined further, such as by iodine scintigram, and it was therefore not possible to determine the origin of the metastasis. It was thought that the metastasis was from thyroid cancer, prostate cancer, or lung cancer that infiltrated the superior vena cava resulting in superior vena cava syndrome, and invasion of the cardiac sac. He received external irradiation $62 \mathrm{~Gy}$ to the neck and chest as a palliative treatment. This treatment decreased the mediastinum lymph node metastasis and cardiac fluid retention and, moreover, improved the edema of the face and fingers. However, one month after the end of radiotherapy, he died due to an increase in heart failure.

\section{Discussion}

As a clinical symptom of SVCS, facial edema and erythema are the most common, as seen in this case. In our case, PET-CT and contrast CT showed enlarged mediastinum lymph nodes infiltrating SVC diagnosed as SVCS. Stracka C, et al. [4] reviewed images of SVCS as follows. Chest image is an important diagonstic tool that can frequently be used to find the abnormality underlying SVCS. $84 \%$ of SVCS patients have abnormal chest X-rays, with $64 \%$ demonstrating widening of the superior mediastinum and $26 \%$ demonstrating pleural effusion. CT and MRI studies are commonly used in the initial evaluation of SVCS and can reveal SVC blockage prior to the development of symptoms. After cancer treatment and when following patients with cancercarrier conditions, it is necessary to keep in mind the possibility of SVCS due to longitudinal lymph node metastasis, as well as chest and mediastinum tumors if rapid facial edema appears [2].

Concerning SVCS therapies, many of the causable diseases of SVCS are malignant tumors. Radiotherapy, chemotherapy with anticancer drugs, and single or combined surgical therapy are usually selected [2,5,6]. Liu H, et al. [7] reported that, for SVCS caused by malignant tumors, percutaneous endoluminal stent therapy could quickly and effectively relieve the clinical symptoms of patients, and the incidence of complications was low. Nanbu H, et al. [2] described that, when chemotherapy or radiotherapy was not available, intravascular stenting was effective in preventing SVC occlusion. In addition, as other symptomatic therapies, he described that the administration of diuretics and corticosteroid drugs may be useful for the prevention of edema.

Raising the head together with oxygen inhalation could reduce the venous pressure. It was desirable to administer the infusion from the lower extremities in order to avoid the upper limbs. Radiation therapy has long been considered a mainstay of SVCS treatment [4]. Some hospitals $[1,2]$ reported that radiation therapy could be effective in cases in which the symptoms needed to be relieved urgently when the general condition was poor, as in our case. Straka C, et al. [4] described that hypo-fractionated RT had the potential to be a more convenient therapy for patients and may provide equal or superior control of underlying malignancies. RT might be combined with stenting and/ or chemotherapy to provide both immediate symptom palliation and long-term disease control. Clinicians should tailor the therapy on a case-by-case basis. Multi-disciplinary care can maximize treatment expediency and efficacy.

Our patient was in a poor general condition. Therefore, he received external irradiation $62 \mathrm{~Gy}$ to the neck and chest as a palliative treatment. This treatment decreased the mediastinum lymph node metastasis and cardiac fluid retention and, moreover, improved the edema of the face and fingers. However, one month after the end of radiotherapy, he died due to an increase in heart failure.

The prognosis of SCVS due to malignant tumors is not good. Wakeda $\mathrm{H}$, et al. [5] discussed about the treatment of SVCS as follows. Management for SVCS associated with a malignant condition involves both treatment of the cancer and relief of the obstructive symptoms. The prognosis for patients with such a condition is poor and median life expectancy is approximately 6 months. Survival among such patients varies and some patients achieve cure of both SVCS and cancer. Bellefqih S, et al. [6] suggested that management of SVCS required a multidisciplinary team. Therapeutic approaches included radiotherapy, chemotherapy and endovascular approach, and the choice of therapy will depend on the severity of the symptoms, the type and the stage of the tumor, but also the patient's general condition.

\section{Conclusion}

We presented a case of SVCS due to mediastinum lymph node 


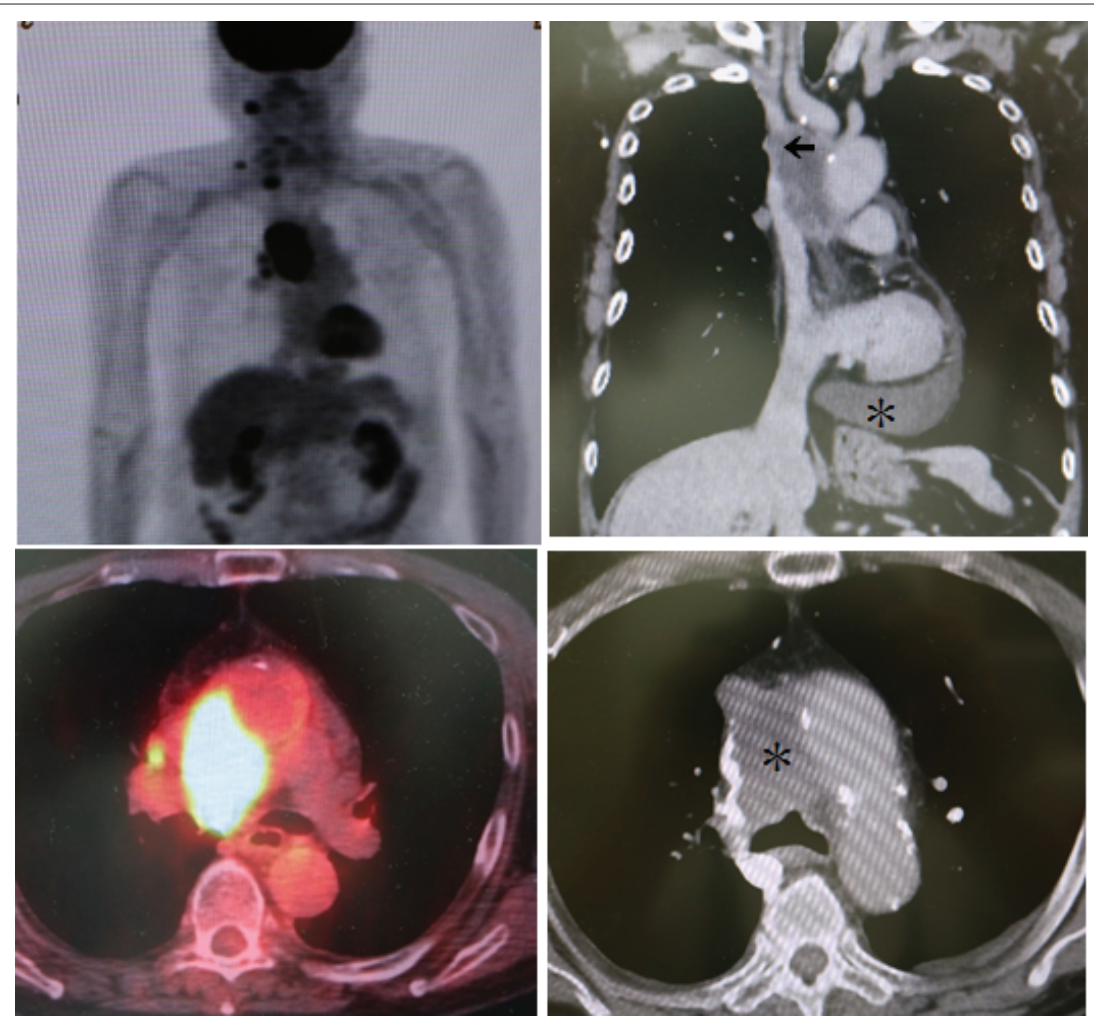

Figure 2: Left, PET-CT showed right cervical and mediastinum lymph node metastasis. Right, the contract enhanced CT showed that the septal lymph node metastasis infiltrated the upper vena cava $(\leftarrow)$. In addition, cardiac fluid retention was observed $(*)$.

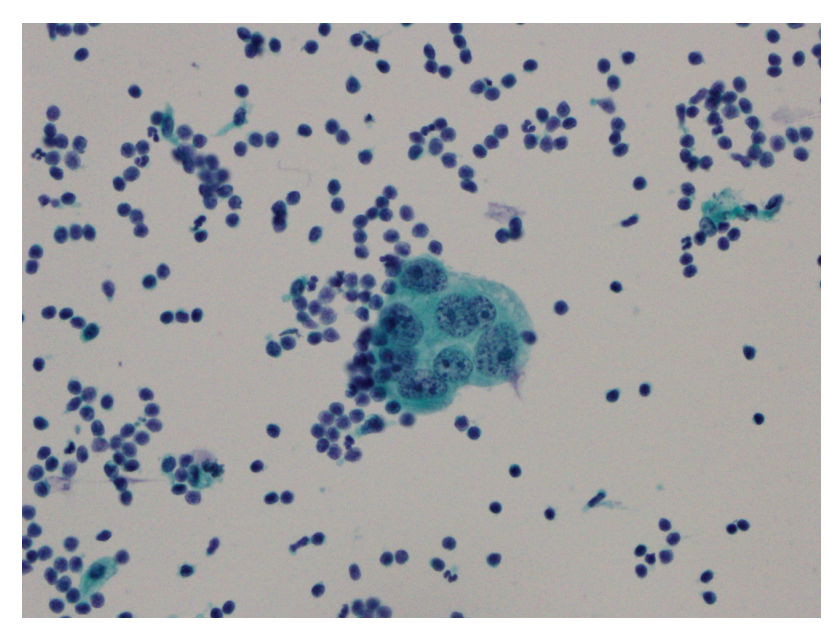

Figure 3: Adenocarcinoma was observed in the cardiac sac fluid by fine needle aspiration cytological examination. In the papanicolaou staining (X400), large atypical cells showed uneven nuclear distribution, irregularities and chromatin elevation.

metastasis. This patient (83-years-old, male, Japanese) had received follow-up for a lung tumor, hormone therapy for prostate cancer, and postoperative cervical lymph node metastasis of the thyroid cancer. Two weeks before, he noticed edema of the face and fingers. PET-CT and contrast enhanced CT were useful for SVCS diagnosis and could show enlarged mediastinum lymph nodes infiltrating SVC, moreover, cardiac fluid retention and pleural fluid. After cancer treatment or following patients with cancer-carrier conditions, it is necessary to keep in mind the possibility of SVCS due to longitudinal lymph node metastasis, as well as chest and mediastinum tumors, if edema of the face and upper limbs appears.

\section{References}

1. Nishio K, Nemoto K, Ishizaki H (1991) Superior vena cave syndrome. Skin Research 33: 343-346.

2. Nanbu $H$, Tanaka A, Tsujiuchi $M$, Aakashiba $T$, Shinohara $H$, et al. (2009) A case of carcinoma of the lower lip with superior vena cava syndrome due to secndary mediastinal lymph node metastasis. Japan J Oral Maxillofac Surg 11: 585-589.

3. Karakhanina WK, Karakhanian WZ, Belczak SQ (2019) Superior vena cave syndrome: endovasular management. J Vasc Bras 18: e20180062.

4. Straka C, Ying J, Kong FM, Willey CD, Kaminski J, et al. (2016) Review of evolving etiologies, implications and treatment strategies for the superior vena cava syndrome. Springerplus 5: 229.

5. Wakeda H, Hamasuna R, Asada Y, KamotoT (2013) Superior vena cava syndrome due to metadtasis from urothelial cancer: A case report and literature review. Urol Ann 5: 291-293.

6. Bellefqih S, Khalil J, Mezouri I, Afif M, Elmaiiaoui S, et al. (2014) Superior vena syndrome with malignanat causes Rev Pneumol Clin 70: 343-352.

7. Liu H, Li Y, Wang Y, Yan L, Zhou P, et al. (2021) Percutaneous transluminal stenting for superior vena cava syndrome caused by malignant tumors: a single-center retrospective study. J Cardiothorac Surg 16: 39 